\title{
DISSIPATION INEQUALITIES IN SYSTEMS THEORY: AN INTRODUCTION AND RECENT RESULTS
}

\author{
Frank Allgöwer \\ Institute for Systems Theory and Automatic Control, University of Stuttgart \\ Pfaffenwaldring 9, 70550 Stuttgart, Germany \\ allgower@ist.uni-stuttgart.de
}

Lyapunov function techniques have received constantly high interest in applied mathematics and in particular in systems and control theory $[1,2,3]$ over the last hundred years. The main reasons for this interest are simplicity, intuitive appeal, and universality of these techniques. Today, there is no doubt that Lyapunov functions techniques are the main tools to be used when one is faced with a stability or stabilization problem. In the analysis and design of control systems, however, there are usually other important requirements besides stability which have to be taken into account. Therefore, it is natural to ask the following question: Is it possible to generalize the ideas of Lyapunov function techniques in order to address for example robustness and performance issues in control systems? Such a generalization is indeed possible and has lead to the powerful concept of dissipativity and dissipation inequalities. Dissipativity has been introduced by Willems [4] and is motivated by the concept of passivity, a paradigm from electrical network theory which relates the stored energy in an electrical network with the supplied energy into the network. Alternatively, one can say that the basic idea behind dissipativity is to generalize the concept of Lyapunov functions techniques to systems with inputs and outputs. Over the past decades, dissipativity turned out to be an extremely useful concept in systems and control theory with plenty of applications in theory and practice.

Like in Lyapunov theory the biggest problem in applications is the construction of a storage function, which is the generalization of the Lyapunov function. However for the important class of polynominal systems, i.e. systems with polynomial nonlinearities, recent advances in the area of computational semialgebraic geometry, namely semidefinite programming and the sum of squares decomposition, allow a reliable and efficient solution in many cases [6, 5].

In this talk we will give a brief historical perspective and an introduction to the system theoretic concept of dissipation inequalities. We will present exemplary recent results on the stability analysis of nonlinear differential algebraic equation systems, minimum phase analysis, and nonlinear feedback and observer design that are based on novel dissipation inequalities and will discuss questions concerning the computation of the storage functions. The methods will be demonstrated and critically assessed with various examples from engineering and systems biology.

\section{References}

1. Lyapunov A. M. The General Problem of the Stability of Motion. CRC Press, 1992.

2. Yoshizawa T. Stability theory by Liapunov's second method. Math. Soc. Japan Tokyo, 1966.

3. Hahn W. Stability of Motion. Springer, 1967.

4. Willems J. C. Dissipative dynamical systems - Part I: General theory // Arch. Ratl. Mech. and Analysis. 1972. V. 45. P. 321-351.

5. Ebenbauer C., Allgöwer F. A dissipation inequality for the minimum phase property // IEEE Transactions on Automatic Control. 2008. V.53. February.

6. P.A. Parrilo Structured Semidefinite Programs and Semialgebraic Geometry Methods in Robustness and Optimization //PhD thesis, California Institute of Technology. 2000. 